\title{
Transseptal catheterization via right subclavian vein
}

\author{
E. J. Epstein and N. Coulshed \\ From the Liverpool Regional Cardiac Centre, Sefton General Hospital, Liverpool 15
}

\begin{abstract}
A method of transseptal catheterization of the left side of the heart via the right subclavian vein has been described. It was attempted in 29 patients and the left atrium was entered in 26 patients. The left ventricle was entered in 21 of the 23 patients ( $91 \%$ ) where entry was attempted.

The risks and advantages of this technique have been discussed. It is a useful additional method for catheterization of the left side of the heart.
\end{abstract}

Transseptal left heart catheterization via the right saphenofemoral route was introduced by Cope (1959) and Ross (1959) in the U.S.A., and first used in this country by Nixon (1960). The original technique was improved by a number of modifications. These included the passage of a large bore catheter over the transseptal puncture needle to enter the left atrium and left ventricle (Brockenbrough and Braunwald, 1960); the fitting of a smaller sized needle tip to the Ross needle to lessen the risk of inadvertent damage (Brockenbrough, Braunwald, and Ross, I962 ; Rainbow, Raftery, and Oakley, 1967); and the use of percutaneous femoral vein puncture to enter the right side of the heart (Cope, 1959; Brockenbrough and Braunwald, 1960; Bevegård, Jonsson, and Karlöf, 1961 ; Endrys and Steinhart, 1962). The method proved to be a relatively easy and safe way of measuring leftsided heart pressures and also for performing left-sided angiocardiography (Brockenbrough et al., 1962; Nixon and Ikram, 1966).

In 1960, Bevegård et al. described a method of transseptal puncture via the right external jugular vein using a special puncture needle. Loskot, Michaljanič, and Musil (1965) described a modification of this technique for left heart catheterization by direct puncture of the right subclavian vein using a shortened Ross transseptal needle.

Percutaneous puncture of the subclavian veins has been used for the insertion of catheters for intravenous infusions, for the measurement of central venous pressure, and more recently for cardiac pacing. Either the subclavicular route (Aubaniac, 1952; Ash-

Received 8 October 1970. baugh and Thomson, 1963; Davidson, BenHur, and Nathen, 1963) or the supraclavicular route (Yoffa, I965) can be used. Our experience in the Liverpool Regional Cardiac Centre relates mainly to the use of supraclavicular subclavian vein puncture for the insertion of emergency pacing catheters in patients with heart block (Macauley and Wright, 1970). The technique has also been used for intravenous infusions and for measurement of central venous pressure. It has been performed in more than 100 patients and the technique evolved has been adapted for the purposes of transseptal puncture.

The purpose of this paper is to describe a method of transseptal catheterization of the left side of the heart using a neck approach through the right subclavian vein. This technique was attempted in 29 patients undergoing routine right and left heart catheterization for assessment of various types of cardiac disorder.

\section{Anatomy of subclavian vein}

The subclavian vein begins at the outer border of the first rib across which it arches, and terminates behind the lower border of the sternoclavicular joint by joining the internal jugular vein to form the innominate vein (Fig. I). The vein is separated from the skin anteriorly throughout its entire course by the clavicle. It is separated from the subclavian artery behind by the scalenus anterior muscle and superiorly lies the cervical fascia, the platysma muscle, and the clavicular head of the sternomastoid muscle. The subclavian vein lies at a slightly lower level on the right side than on the left, and the junction of the subclavian and internal jugular veins may be inferior to the right sternoclavicular joint. A puncture needle must therefore be inserted further, and at a steeper angle on the right side to enter the subclavian vein. 


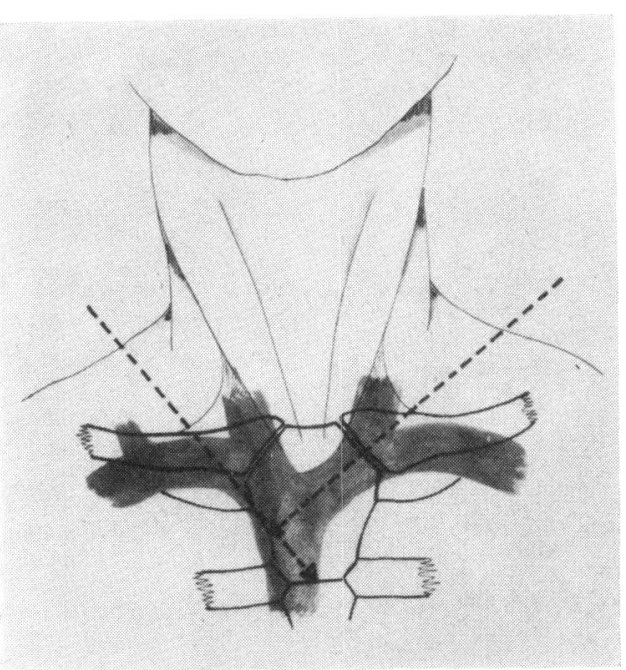

FIG. I Relation of the subclavian veins to surface anatomy. The dotted line show the direction of the puncture needle to enter the subclavian vein. The subclavian vein lies below the upper border of the clavicle. The internal jugular and subclavian veins join behind the sternoclavicular joint.

\section{Equipment}

This is shown in Fig. 2 and consists of the following. (I) A Seldinger type puncture needle with a No. 18 size cannula; (2) an $80 \mathrm{~cm}$ length 0.035 in flexible spring guide wire; (3) a size No. 6 U.S.C.I. catheter introducer with a central dummy catheter and an outer Teflon sheath; (4) an $80 \mathrm{~cm}$ length 0.045 in flexible spring guide wire; (5) a radioopaque yellow Kifa type disposable polyethylene catheter, $39.5 \mathrm{~cm}$ in length with the terminal $3 \mathrm{~cm}$ curved into a ' $\mathrm{J}$ ' shape and with three pairs of parallel side-holes; and (6) a short No. 17 gauge Ross type of transseptal puncture needle, length $4 \mathrm{r} \mathrm{cm}$. The whole shaft of the needle is slightly curved with the terminal 2 to $3 \mathrm{~cm}$ curved more sharply to a radius of $7 \mathrm{~cm}$.

\section{Technique}

The patient lies flat, with a pillow under the shoulders to extend the neck slightly. The junction of the clavicular head of the right sternomastoid with the clavicle - the clavisternomastoid angle - is identified by palpation, if necessary after tensing the muscle by raising the head against resistance, and the skin marked with blue dye. In addition, the right sternoclavicular joint and the mid-point of the sternal angle are also marked. The skin is cleaned and towelled and local anaesthetic is injected about $\mathrm{I} \mathrm{cm}$ above the clavisternomastoid angle. A small nick is made in the skin with a scalpel and the platysma and fascia are spread open with fine artery forceps. The puncture needle is introduced and directed downwards, forwards, and medially towards the centre of the sternal angle. The needle usually enters the vein at a distance of 2 to $4 \mathrm{~cm}$ from the skin. A I $\mathrm{ml}$ syringe attached to the needle allows a free flow of blood when the vein is entered. If the vein is not entered immediately then the needle is reinserted and directed more laterally towards the second costosternal junction. The fine $80 \mathrm{~cm}$ $(0.035$ in) flexible guide wire is passed through the cannula to the right atrium under fluoroscopic control. The cannula is removed and replaced by the U.S.C.I. catheter introducer consisting of the dummy catheter and outer Teflon sheath. The fine guide wire and dummy catheter are removed and replaced by the thick $80 \mathrm{~cm}(0.045$ in) flexible guide wire. The Teflon sheath is removed and the radio-opaque catheter passed over the guide wire to the right atrium. The guide wire is then withdrawn.

Alternatively we have performed the initial subclavian vein puncture with a large No. I6 gauge puncture needle which will take the thick guide wire direct and thus eliminate the use of the U.S.C.I. catheter introducer. Usually we have preferred to use the finer needle for the initial puncture in case of inadvertent entry into the subclavian artery.

The modified $4 \mathrm{I} \mathrm{cm}$ Ross needle is passed down the catheter until the tip is just within the end and then it is connected to the pressure manometer and the pressure tracing displayed on a cathode ray oscilloscope screen. The needle is rotated in a clockwise direction (looking down the shaft of the needle), to avoid entering the right atrial appendage, until it is pointing medially between 7 and 8 o'clock. It is then gently pulled from below upwards along the atrial septum until the fossa ovale is engaged. If the fossa ovale cannot be felt then the middle third of the medial wall of the right atrium is taken as the site for puncture. After engaging the septum it is punctured by advancing the needle sharply beyond the tip of the catheter as for transseptal puncture via the saphenofemoral route. Pressure is continuously monitored throughout the procedure.

As soon as the left atrium is entered the catheter is advanced over the needle for about 2 to $3 \mathrm{~cm}$ and the needle is rotated in a counterclockwise direction to bring the catheter against the septal wall of the atrium. The catheter is then advanced into the left ventricle (Fig. 3). Occasionally further manipulation and even the use of a spring guide wire is necessary to facilitate entry into the left ventricle. The needle is then withdrawn and the catheter connected to the pressure transducer.

When the study has been completed the catheter is withdrawn and the head and shoulders slightly raised to prevent bleeding from the puncture site.

\section{Results}

These are shown in the Table. Transseptal puncture was attempted in 29 patients. The left atrium was entered in 26 and the left ventricle in 21 patients. No attempt was made to enter the left ventricle in 3 patients and 


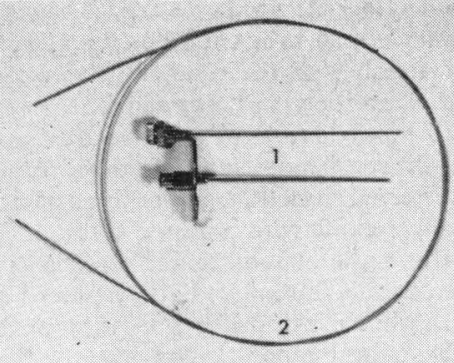

3

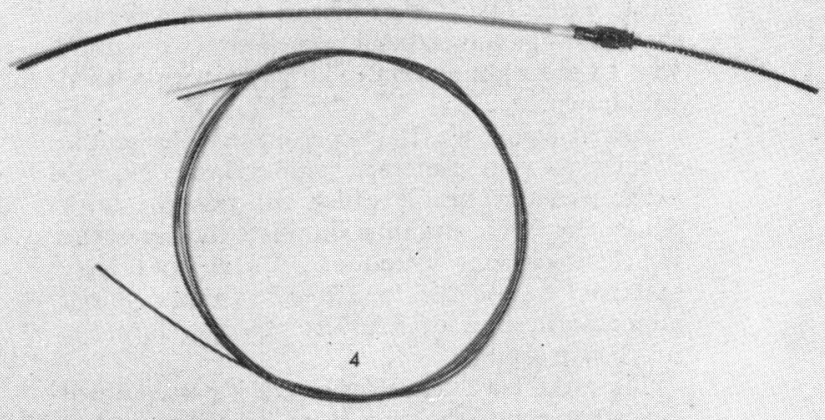

5

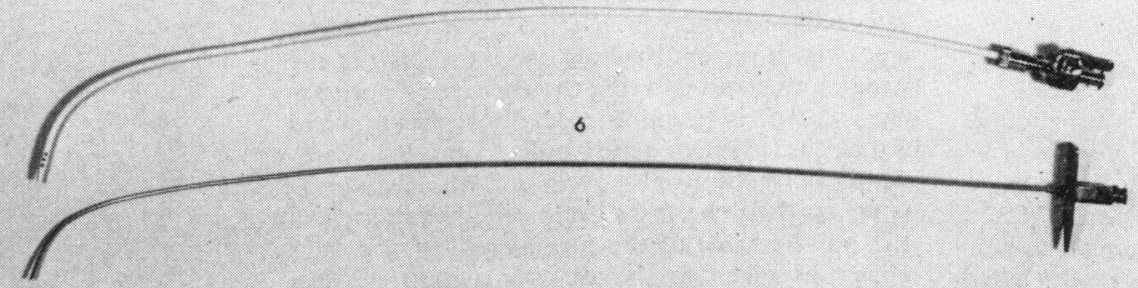

FIG. 2 Transseptal puncture equipment. I, No. I8 gauge Seldinger puncture needle; $2,80 \mathrm{~cm}$ 0.035 in flexible guide wire; 3, No. 6 gauge U.S.C.I. catheter introducer with central dummy catheter and outer Teflon sheath; 4, $80 \mathrm{~cm} 0.045$ in flexible guide wire; 5, Radio-opaque $39.5 \mathrm{~cm}$ polyethylene catheter; 6 , Ross transseptal needle $41 \mathrm{~cm}$ length with curved shaft and sharper tip curvature.

attempts at entry failed in 2 other patients. Thus, the left ventricle was catheterized in 21 out of 23 attempts, a success rate of 91 per cent. Angiocardiography was performed through the neck transseptal catheter in 5 patients using a Straube end-hole occluder assembly (U.S. Catheter Corporation). In 2 of the patients the dye was injected into the left atrium and in the other 3 patients into the left ventricle (Fig. 4).

There were no significant complications in any of the 29 patients. A few patients with raised venous pressure had minor haematoma formation at the needle puncture site.

\section{Discussion}

The technique of percutaneous puncture of the subclavian vein was developed on this unit after careful assessment of the anatomy of the vein by dissection in cadavers and angiographic studies in patients (Macauley and Wright, 1970).

The risks of subclavian vein puncture include pneumothorax from puncture of the apical pleura and subclavian artery puncture. Both are due to insertion of the needle too far posteriorly and may be avoided by forward angulation of the needle during the puncture. Air embolism can occur if the venous pres- 
sure is very low but is unlikely with the patient recumbent and if care is taken to occlude the cannula with the thumb before inserting the guide wire (Yoffa, 1965; Flanagan et al., 1969). Bleeding after removal of the catheter will cease when the patient is propped up so that the puncture site lies above the level of the venous column. With a very high venous pressure there may be slight haematoma formation at the insertion site in the neck. Subclavian vein thrombosis has not developed in the patients reported here nor has it been reported (Yoffa, I965; Loskot et al., 1965).

There may be difficulty in engaging the atrial septum from above and the curve of the tip of the Ross transseptal needle needs to be more pronounced than for transseptal puncture via the femoral route. The Ross type of needle is used routinely in this unit for conventional transseptal puncture, and for this reason we have generally used a short modified Ross needle for transseptal puncture via the subclavian vein. As an alternative we have also used a shortened version of the Hammersmith modification of the Brockenbrough needle (Rainbow et al., 1967) and found it equally satisfactory.

The risks are the same as for a conventional transseptal puncture and include aortic puncture, pericardial entry, haemopericardium, and systemic embolism from dislodgement of left atrial thrombus. None of these complications has occurred in this series of patients. Since the needle is short and there is little tension on the great veins, the procedure causes less discomfort than transseptal puncture from below.

Once the left atrium is entered it is relatively easy to catheterize the left ventricle since the catheter advances naturally towards the orifice of the mitral valve. The success rate for left ventricular entry was 21 out of 23 attempts, i.e. 9r per cent (Table). This is at least comparable to the frequency of left ventricular entry by the conventional transseptal puncture technique.

Selective left ventricular or left atrial angiocardiography can be performed through the catheter with the use of a Straube end-

TABLE Transseptal catheterization via right subclavian vein in 29 patients

\begin{tabular}{lll}
\hline & $\begin{array}{l}\text { Left atrial } \\
\text { catheterization }\end{array}$ & $\begin{array}{l}\text { Left ventricular } \\
\text { catheterization }\end{array}$ \\
\hline Attempted & 29 & 23 \\
Failed & 3 & 2 \\
Succeeded & $26(89.5 \%)$ & $21(91 \%)$ \\
\hline
\end{tabular}

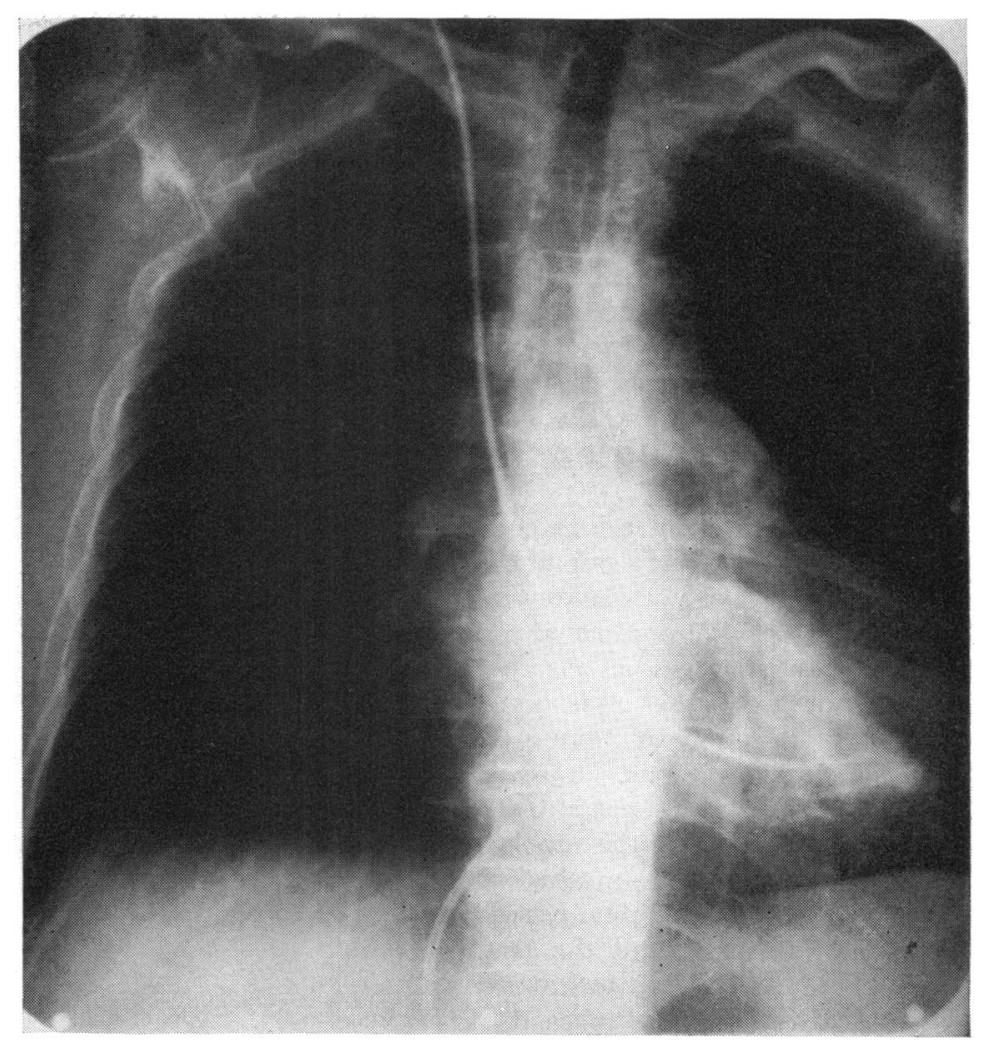

FIG. 3 Transseptal catheter in situ. The catheter passes from the right subclavian vein to the right atrium and across the atrial septum to enter the left atrium and then the left ventricle.

hole occluder assembly (Fig. 4). Because of the relatively straight entry into the left ventricle, the catheter has less tendency to recoil into the left atrium during injection of contrast medium than with transseptal catheters inserted through the femoral vein. The subclavian route for left heart catheterization leaves the legs unrestricted so that exercise studies can easily be performed. If simultaneous arterial catheterization is required the brachial arterial route can be used so that the legs still remain free.

In some patients the saphenofemoral route for transseptal puncture may be undesirable or not possible. If there has been a previous disorder of the veins in the pelvis or right leg such as an ileofemoral vein obstruction or there are severe varicose veins, then manipulation of catheters in the leg veins is contraindicated. When there is a lumbosacral spinal deformity it may be impossible to manipulate a transseptal needle over the pelvic brim. Arteriosclerotic iliac arteries distort the vein 
and may also prevent the passage of the transseptal needle through the pelvis. In some patients the tension of the needle on the iliac veins and inferior vena cava may cause great pain and prevent its advancement or manipulation. With massive right atrial enlargement usually due to severe tricuspid valve disease, there is distortion of the inferior vena cava, which alters the alignment of the transseptal needle and prevents it from engaging the septum. In such patients the superior vena cava does not seem to be distorted and the septum can usually be engaged from above via the subclavian vein.

There have been no serious complications using this technique and our findings are in agreement with those of Loskot et al. (1965). In this unit we have performed nearly 1,000 transseptal catheter studies via the saphenofemoral route over a period of 9 years. There has been a failure rate of about 4 per cent for a multiplicity of reasons. In some of these patients we have later successfully performed transseptal puncture via the subclavian vein.

Most of the patients in this series were selected because of difficulties in routine transseptal puncture, and the results could clearly be improved if the technique was used routinely as a first choice method. Nevertheless, in the last I I patients the left atrium was entered in 9 patients and the left ventricle in 8 patients, a success rate at least comparable to that for conventional transseptal catheterization.

This experience is relatively limited and the method may be improved by further modifications. For example, the tip of the polyethylene catheter could be moulded into a ' $U$ ' shape to allow entry into the aorta from the left ventricle. For this purpose it may be necessary to use a needle and catheter of up to 50 $\mathrm{cm}$ in length. Alternatively the short polyethylene catheter could be replaced by a Cournand or Gensini type of catheter with the aid of a long flexible guide wire, after the left atrium has been entered (Verel, 1967).

This technique is a useful addition to other well-established methods of left heart catheterization. It is certainly a little more difficult to perform than the standard method via the femoral vein, and we do not suggest that it should supplant that procedure. However, in certain special circumstances, as outlined above, transseptal puncture via the subclavian vein may have considerable advantages and it may be the only available route for direct entry into the left atrium.

We wish to thank Sister J. Davies, in charge of the catheter laboratory and Mrs B. Lea, senior

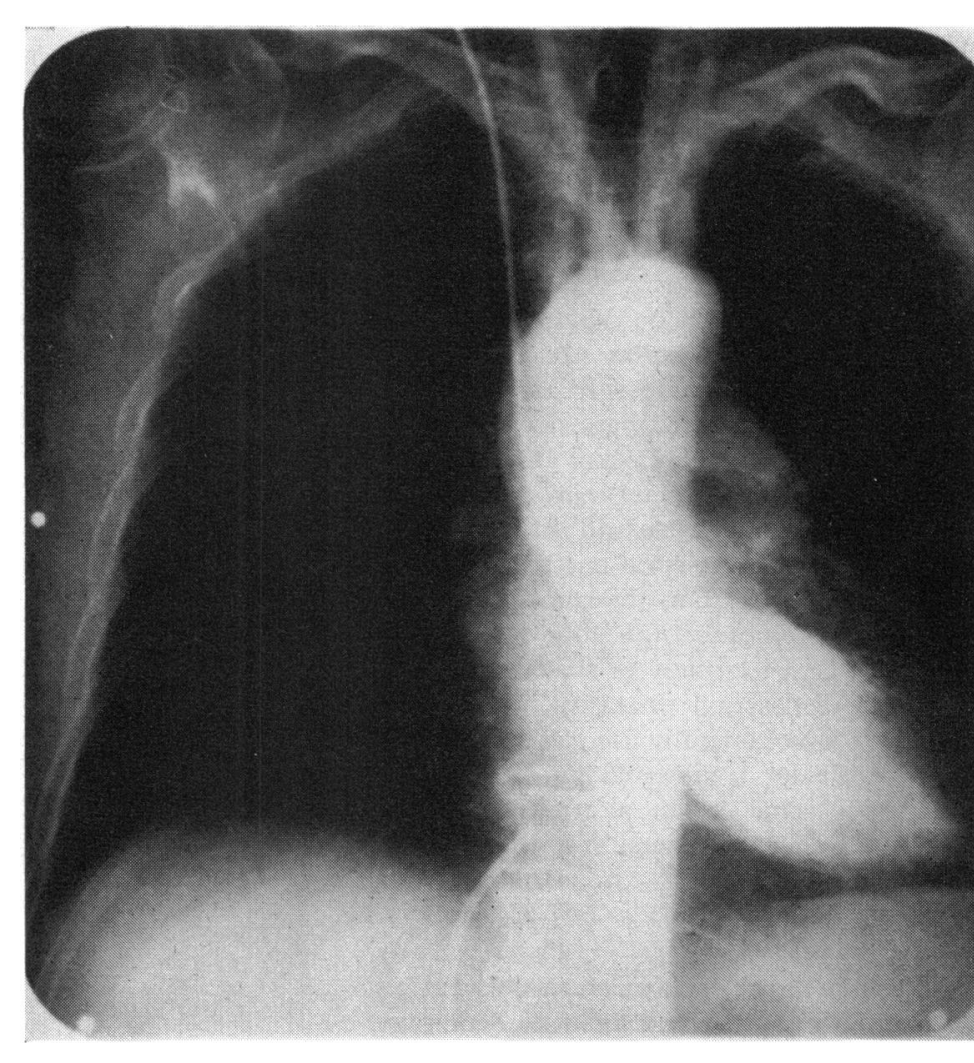

FIG. 4 Left ventriculogram; Posteroanterior view; systolic exposure. Same patient as in Fig. 3. Radio-opaque dye has been injected into the left ventricle via the transseptal catheter using a Straube end-hole occluder. The left ventricular cavity and aorta are outlined. There was no mitral regurgitation.

cardiographic technician, and their respective staffs for invaluable technical assistance.

\section{References}

Ashbaugh, D., and Thomson, J. W. W. (1963). Subclavian-vein infusion. Lancet, 2, 1138.

Aubaniac, R. (1952). L'injection intraveineuse sousclaviculaire. Avantages et technique. Presse Médicale, 60, 1456.

Bevegård, S., Carlens, E., Jonsson, B., and Karlöf, I. (1960). A technique for transseptal left heart catheterization via the right external jugular vein. Thorax, 15, 299.

Bevegård, S., Jonsson, B., and Karlöf, I. (I96I). Percutaneous technique for transseptal left heart catheterization via the right femoral vein. Scandinavian fournal of Clinical and Laboratory Investigation, 13, 439.

Brockenbrough, E. C., and Braunwald, E. (1960). A new technic for left ventricular angiocardiography and transseptal left heart catheterization. American fournal of Cardiology, 6, 1062.

Brockenbrough, E. C., Braunwald, E., and Ross, J., 
Jr. (I962). Transseptal left heart catheterization. A review of 450 studies and description of an improved technic. Circulation, 25, 15.

Cope, C. (1959). Technique for transseptal catheterization of the left atrium: preliminary report. Fournal of Thoracic and Cardiovascular Surgery, 37, 482.

Davidson, J. T., Ben-Hur, N., and Nathen, H. (1963). Subclavian venepuncture. Lancet, 2, II 39.

Endrys, J., and Steinhart, L. (1962). Transseptal catheterization and cardioangiography of the left heart by a percutaneous route through the femoral vein. Cardiologia, 4I, 47.

Flanagan, J. P., Gradisar, I. A., Gross, R. J., and Kelly, T. R. (1969). Air embolus - a lethal complication of subclavian venipuncture. New England Fournal of Medicine, 281, 488.

Loskot, F., Michaljanič, A., and Musil, J. (1965). Right and left heart catheterization via the subclavian veins. Cardiologia, 46, II4.

Macauley, M. B., and Wright, J. S. (1970). Trans- venous cardiac pacing. Experience of a percutaneous supraclavicular approach. British Medical fournal, 4, 207.

Nixon, P. G. F. (1960). The transeptal approach to the left atrium in mitral regurgitation. Thorax, 15, 225.

Nixon, P. G. F., and Ikram, H. (I966). Left heart catheterization with special reference to the transseptal method. British Heart fournal, 28, 835.

Rainbow, R. L. G., Raftery, E. B., and Oakley, C. M. (1967). Improved design for a transseptal needle. British Heart fournal, 29, 394.

Ross, J., Jr. (1959). Transeptal left heart catheterization: a new method of left atrial puncture. Annals of Surgery, 149, 395.

Verel, D. (1967). Catheterization of the left atrium by Ross needle and Seldinger wire techniques. British Heart fournal, 29, 380.

Yoffa, D. (1965). Supraclavicular subclavian venepuncture and catheterisation. Lancet, 2, 614. 